\title{
IgG isotype profile is correlated with cardiomegaly in Beagle dogs infected with distinct Trypanosoma cruzi strains
}

\author{
Paulo M.M. Guedes ${ }^{\mathrm{a}, \mathrm{c}, *}$, Vanja M. Veloso ${ }^{\mathrm{a}, \mathrm{c}}$, Kenneth J. Gollob ${ }^{\mathrm{b}}$, \\ Luis C.C. Afonso ${ }^{c}$, Ivo S. Caldas ${ }^{c}$, Priscila Vianna ${ }^{\mathrm{b}}$, Marta de Lana ${ }^{\mathrm{d}}$, Egler Chiari ${ }^{\mathrm{a}}$, \\ Maria T. Bahia ${ }^{c}$, Lúcia M.C. Galvão ${ }^{a}$ \\ ${ }^{a}$ Departamento de Parasitologia, Instituto de Ciências Biológicas, Universidade Federal de Minas Gerais, Belo Horizonte, \\ Minas Gerais, Brazil \\ ${ }^{\mathrm{b}}$ Departamento de Bioquímica e Imunologia, Instituto de Ciências Biológicas, Universidade Federal de Minas Gerais, \\ Belo Horizonte, Minas Gerais, Brazil \\ ${ }^{\mathrm{c}}$ Departamento de Ciências Biológicas - NUPEB, Universidade Federal de Ouro Preto, Ouro Preto, Minas Gerais, Brazil \\ ${ }^{\mathrm{d}}$ Escola de Farmácia, Universidade Federal de Ouro Preto, Ouro Preto, Minas Gerais, Brazil \\ Received 13 May 2007; received in revised form 6 March 2008; accepted 14 March 2008
}

\begin{abstract}
A systematic study following infection by various strains of the protozoan parasite, Trypanosoma cruzi, and the simultaneous monitoring of the humoral immune response together with the elicited cellular response, could add greatly to our understanding of differences between strains of this important human pathogen. In that sense, acute and chronic infections with distinct $T$. cruzi strains (Y, Berenice-78 and ABC) in Beagle dogs were studied through a longitudinal evaluation of immunoglobulin $\mathrm{G}$ (IgG), IgG1 and IgG2 isotypes (by ELISA and flow cytometry (FC)), as well as measurements of peripheral blood mononuclear cell (PBMC) proliferation over a 100-week period, and their correlation with cardiomegaly. Our results show that infected animals presenting cardiomegaly showed lower or absent levels of IgG1 during the chronic phase of the infection, when compared to those that did not show an increase in heart weight. In that manner, our results suggest that IgG1 could be used as a marker for cardiac pathogenicity in Chagas disease.
\end{abstract}

(C) 2008 Elsevier B.V. All rights reserved.

Keywords: Trypanosoma cruzi; Chagas disease; Beagle dogs; Immunopathogenesis; IgG isotype

\section{Introduction}

Chagas disease is endemic from Mexico to Argentina, where it is estimated that 13 million people are infected with Trypanosoma cruzi in the Central and

\footnotetext{
* Corresponding author at: Departamento de Ciências Biológicas NUPEB, Laboratório de Doença de Chagas, Universidade Federal de Ouro Preto, Campus Universitário, Bauxita, 35400-000 Ouro Preto, Minas Gerais, Brazil. Tel.: +55 313559 1690; fax: +55 3135591680 .

E-mail address: guedespm@nupeb.ufop.br (P.M.M. Guedes).
}

South America, and 75 million present a potential risk of infection (WHO, 2005). During chronic phase of infection, the majority of chagasic patients (about 70\%) do not present clinical signs (indeterminate form), however, about 10-20\% develop a digestive (megaesophagus and megacolon) and cardiac form of disease, respectively (Tafuri, 1987). The immunopathological mechanisms involved in the pathogenesis of chagasic cardiomyopathy are not completely elucidated. The profile of inflammatory cells, as well as production of cytokines, chemokines, antibodies and soluble factors 
that act in cellular signaling may be the key for a better comprehension of chronic lesions genesis, determining why some patients demonstrate cardiac manifestations and another remain with the indeterminate form of Chagas disease.

A major set back in research efforts to elucidate the pathogenesis mechanisms of chronic Chagas' disease is the lack of a suitable animal model. The canine model reproduces diffused chronic myocarditis and has shown the cardiac, pathological and electrocardiographic changes also seen in humans (Lana et al., 1992; Guedes et al., 2007). Moreover, recent findings demonstrated that the dog is a good model for chemotherapy studies in Chagas' disease (Guedes et al., 2002). The aim of this study is to evaluate the kinetics of IgG1 and IgG2 isotypes production and proliferation of peripheral blood mononuclear cells (PBMC) against parasite antigens, correlating the data to cardiomegaly found in Beagle dogs infected with Y, Berenice-78 and $\mathrm{ABC} T$. cruzi strains.

\section{Materials and methods}

\subsection{T. cruzi strains}

In this study, three distinct strains were used. The Y T. cruzi strain isolated from an acute human case (Silva and Nussenzweig, 1953); Berenice-78, isolated by xenoculture (the technique consist in putting nymphs of not infected haematophagus triatomines insect to feed in a patient and culture is performed with intestinal contents and parasites isolated) in 1978 from what was considered the first clinical case of the disease in Brazil, in a patient with the indeterminate form of the disease (Lana and Chiari, 1986) and the ABC strain, which was also isolated by xenoculture from a patient with heart and digestive manifestations from the Minas Gerais state, Brazil (Brener, 1965). These strains were isolated from the individuals with different clinical forms of Chagas disease and showing distinct patterns of virulence and pathogenicity for vertebrate host (Guedes et al., 2007).

\subsection{Experimental animals and infection}

Sixteen 4-month-old Beagle dogs from the kennel of the Universidade Federal de Ouro Preto (UFOP), Minas Gerais, Brazil were used in this study. All procedures and experimental protocols were conducted in accordance with the COBEA (Brazilian College of Animal Experimentation) and approved by the Ethics Committee in Animal Research of the UFOP. Animals were fed with commercial ration and water ad libitum. Before the study, the animals were dewormed and vaccinated against several infectious diseases. Animals were inoculated with 4000 blood trypomastigotes per $\mathrm{kg}$ of body weight by intra-peritoneal route.

For ELISA, flow cytometry and PBMC proliferative assays, blood or serum samples were collected from dogs at regular interval of 2 and 10 weeks during the acute and chronic phases, respectively.

\subsection{Peripheral blood mononuclear cells proliferative assay}

Peripheral blood mononuclear cells (PBMC) were isolated by Ficoll-Hipaque density gradient centrifugation and washed three times with RPMI (GIBCO, Grand Island, New York, USA). The cells $\left(3.5 \times 10^{6}\right)$ were stimulated with parasite antigens $\left(5 \times 10^{6}\right.$ trypomastigotes $/ \mathrm{ml}$ ) or Concanavalin-A mitogen $(4 \mu \mathrm{g} / \mathrm{ml})$, in a final volume of $200 \mu \mathrm{l}$. Cells were then incubated for three and five days at $37{ }^{\circ} \mathrm{C}$ in a $5 \%$ $\mathrm{CO}_{2}$ atmosphere with Concanavalin- $\mathrm{A}$ and trypomastigote antigen, respectively. Cultures were pulsed with $0.2 \mu \mathrm{Ci} /$ well of $\left[{ }^{3} \mathrm{H}\right]$-thymidine in the last $16 \mathrm{~h}$ of incubation, and processed for scintillation counting. Results of stimulated and non-stimulated PBMC cultures were expressed in CPM (counts per minute).

\subsection{Conventional serology (ELISA)}

Serum samples were stored at $-80{ }^{\circ} \mathrm{C}$ and ELISA tests were performed according to Voller et al. (1976). Briefly, ELISA plates were sensitized with $T$. cruzi antigen prepared by alkaline extraction of $\mathrm{Y}$ strain, obtained at exponential growth in LIT medium. The sera were added, and the antibody binding was detected by using peroxidase-labeled anti-dog IgG. Total IgG or IgG1 and IgG2 isotypes conjugated to horseradish peroxidase (Bethyl Laboratories, Montgomery, USA) were used for the determination of antibody levels. The plates were read with a spectrophotometer at $490 \mathrm{~nm}$ filter (BIO-RAD, 3550). Cut-off was determined using the absorbance mean of ten uninfected animals plus two standard deviations.

\subsection{Anti-live trypomastigote antibodies (FC, flow cytometry)}

FC analysis was performed as described by MartinsFilho et al. (1995) and modified for plates of 96 wells with U bottom (LINBRO, ICN Biomedicals, OH, USA), except that canine sera (diluted 1:128) were used instead of human sera and fluorescein isothiocyanate 
(FITC)-labeled mouse anti-dog total IgG, IgG1 and IgG2 (1:400 dilution; Bethyl Laboratories, Montgomery, USA) substituted FITC-labeled anti-human IgG. Each assay included negative and positive controls (internal control of non-specific binding in which parasites were incubated with FITC-conjugated antibodies alone, or parasite incubated with serum and anti mouse IgG FITC-conjugated, and sample known positive). For each assay, 50,000 events were analyzed and the trypomastigotes were identified and selectively gated on the basis of their specific forward and side light-scattering properties. Data were expressed as the relative percentage of fluorescent parasites (PFP). Cytofluorometric acquisition was performed by FACScan (Becton Dickinson, San Jose, CA, USA), and the CellQuest software package was used for both flow cytometry data storage and analysis.
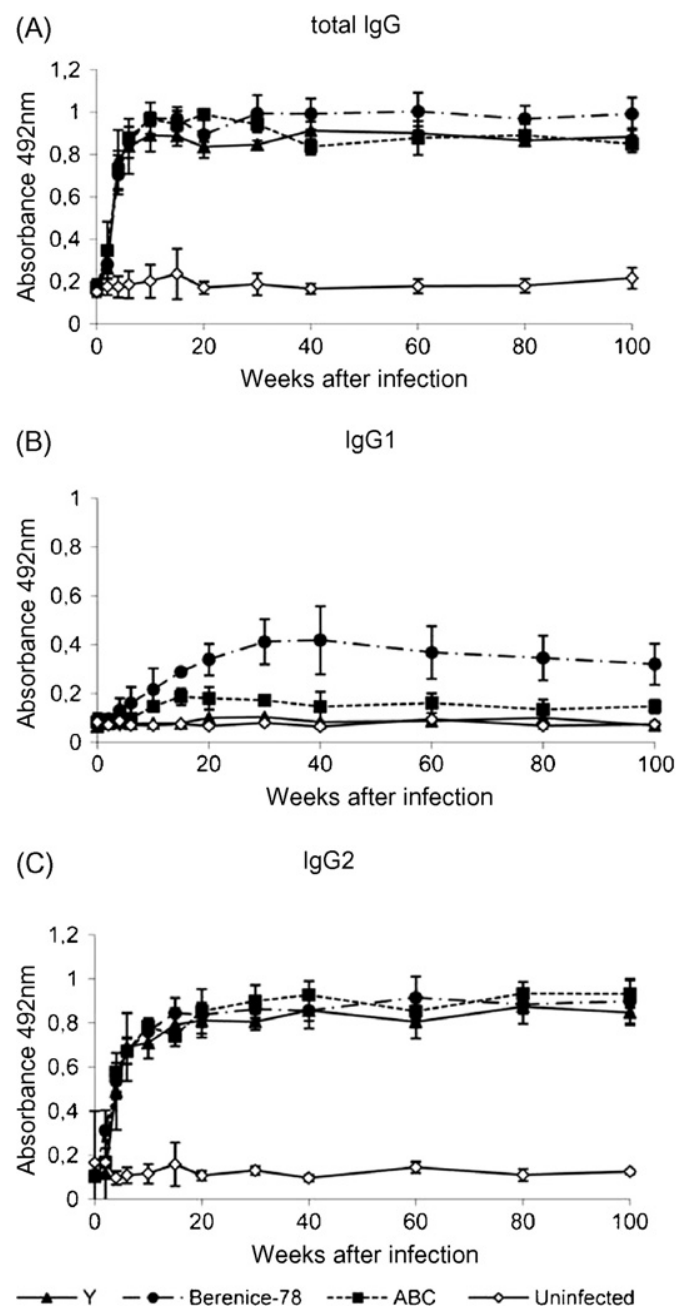

\subsection{Cardiomegaly}

All animals were euthanized by injection with thionembutal (Abbott, São Paulo, Brasil) $0.5 \mathrm{ml} / \mathrm{kg}$ of body weight $(0.03 \mathrm{~g} / \mathrm{ml}$ of saline solution $0.8 \%) 100$ weeks after infection. Necropsy was performed and heart and animal weights were determined. The cardiomegaly was evaluated by determining a heart index (heart weight/total body weight $\times 100$ ) and also by inspection for gross alterations. Cardiomegaly was considered in the infected animals when the heart index was significantly greater than observed in uninfected animals.

\subsection{Statistical analysis}

Regression analysis was used to compare antibody levels and PBMC proliferation; regression lines were
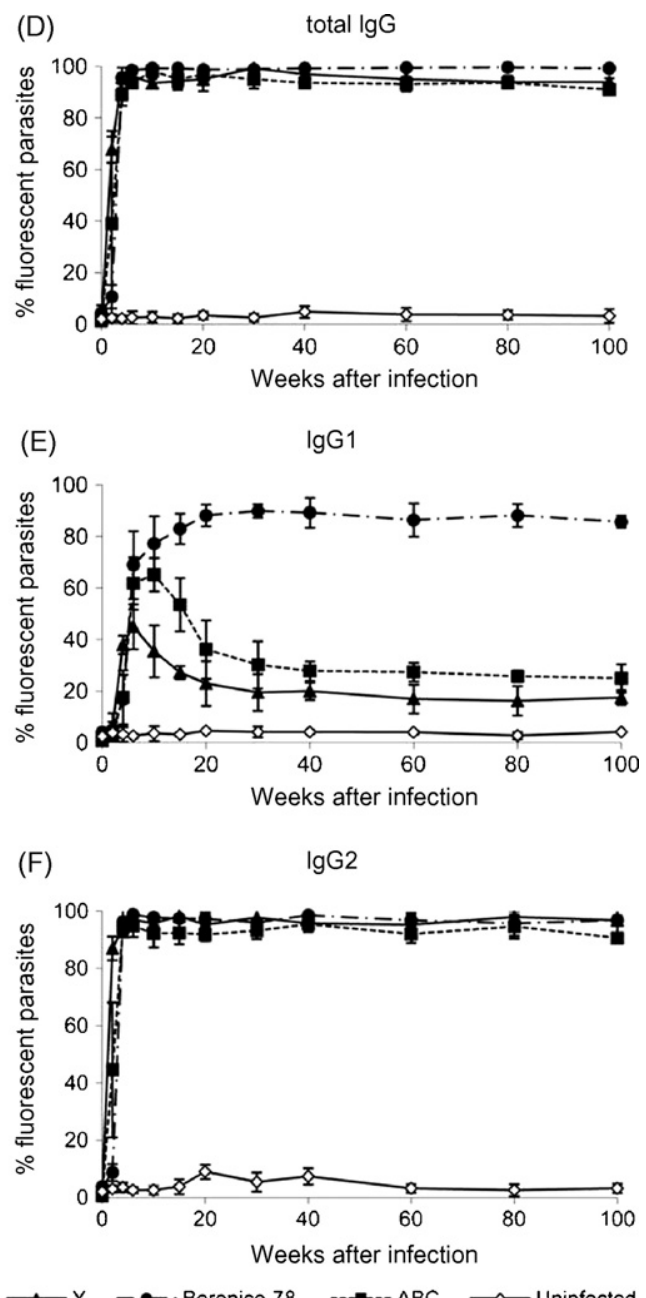

Fig. 1. Trypanosoma cruzi specific IgG antibodies in sera of infected Beagle dogs with Y, Berenice-78 and ABC strains. (A) and (D) levels of IgG total, (B) and (E) levels of $\operatorname{IgG1},(\mathrm{C})$ and (F) levels of $\operatorname{IgG} 2$ in conventional serology (A-C) and flow cytometry (D-F). 
compared by analysis of covariance (Snedecor and Cochran, 1989). The association between antibody levels and the PBMC proliferation was tested by using the Pearson correlation. In all cases, differences were considered statistically significant when $P<0.05$.

\section{Results and discussion}

We initially evaluated the establishment of humoral response kinetics during the acute phase of the disease. We observed the same profile of total $\operatorname{IgG}$ and $\mathrm{IgG} 2$, both by ELISA and FC, in the animals infected with the three $T$. cruzi strains. However, different IgG1 profiles were observed by ELISA and FC (Fig. 1). The distinct production of IgG subclasses by ELISA and FC was described for human patients of different geographical areas (Cerban et al., 1993; Morgan et al., 1996; Cordeiro et al., 2001).

High levels of IgG1 antibodies $(P<0.05)$ by ELISA and FC were detected only in serum of animals infected with Berenice-78 strain during acute and chronic phases of infection. Curiously, no significant increase in IgG1 levels in dogs infected with $\mathrm{Y}$ and $\mathrm{ABC}$ were observed by ELISA (levels were similar to those observed in negative controls) (Fig. 1B and E).

It has been previously suggested, but not proved yet, with the use of commercial antibodies that human patients and dogs control $\operatorname{IgG}$ isotypes production in a similar manner, however, mediated by the production of different cytokines (Kawano et al., 1994; Deplazes et al., 1995). It has been observed that IL-4, IL-5 and IL10 are responsible for the production of IgG1 and IgG3 isotypes (Briere et al., 1994; Reed and Scott, 1993), while IL-12, IFN- $\gamma$, TNF stimulate IgG2 production. On the other hand, different results were obtained using purified monoclonal antibodies (Day, 2007; Quinnell et al., 2003). Despite that research on immunoglobulins is important for the understanding of immunopathological mechanisms, there are controversies about nomenclature, function, regulation and specificity in canine $\operatorname{IgG}$ subclasses.
In an attempt to correlate IgG subclasses and cardiac pathology, we determined cardiomegaly presence in the animals. During the chronic phase of infection only $50 \%$ of Berenice-78 infected animals showed cardiomegaly, while all animals infected with the $\mathrm{Y}$ or the ABC strain showed increase of the heart (Table 1). We also observed heart area flaccidity in $100 \%$ of the animals infected with the $\mathrm{Y}$ and $\mathrm{ABC}$ strains, whereas only $50 \%$ of the animals infected with Berenice- 78 presented similar lesions.

Our results showed that the presence of cardiomegaly in $T$. cruzi-infected dogs was associated with low or absence of IgG1 production during the chronic phase of the disease (Table 1; Fig. 1B and E). Our group observed in this experiment higher cardiac destruction (inflammation and fibrosis) and electrocardiographs alterations in dogs infected with $\mathrm{Y}$ and $\mathrm{ABC}$ strains, than Berenice-78 infected dogs (unpublished data). The results suggest that IgG1 production in dogs is associated with the anti-inflammatory cytokines, our results corroborate previous observations that demonstrated that the increase in heart weight from infected patients is related to the intensity of the inflammatory infiltrate and fibrosis in this organ (Oliveira, 1998). A correlation analysis of specific $T$. cruzi IgG1 antibody levels (ELISA and FC) and cardiomegaly was performed 100 weeks after infection. Our results show that production of IgG1 isotype during the T. cruzi infection of Beagle dogs are correlated to the parasite strains, while IgG1 levels measured by ELISA are inversely correlated with cardiomegaly $(P<0.01$, $R=0.699$ ) (Fig. 2). However, other dog breeds should be analyzed to confirm the findings, maybe different responses could be observed.

The correlation amongst the different clinical forms of Chagas disease and the levels of the IgG isotypes is not clearly defined. Several authors have tried to demonstrate the correlation between IgG isotypes and the severity of the different clinical forms of Chagas disease both in patients (Cerban et al., 1993; Morgan et al., 1996; Michailowsky et al., 2003) and in

Table 1

Correlation between IgG1 production, PBMC proliferation and cardiomegaly in Beagle dogs infected with Y, Berenice-78 and ABC Trypanosoma cruzi strains

\begin{tabular}{lllll}
\hline T. cruzi strains & IgG1 production by & PBMC proliferation 100 & $\begin{array}{l}\text { Number of dogs } \\
\text { weeks after infection (CPM) }\end{array}$ & with cardiomegaly/total \\
\cline { 2 - 3 } & ELISA & Flow cytometry & $7201 \pm 663$ & $(4 / 4)$ \\
Y & Not detected & Acute phase & $(2 / 4)$ \\
Berenice-78 & Acute and chronic phases ${ }^{\mathrm{a}}$ & Acute and chronic phases & $4813 \pm 752$ & $(4 / 4)$ \\
ABC & Not detected & Acute phase & $5957 \pm 982$ & \\
\hline
\end{tabular}

\footnotetext{
${ }^{\text {a }}$ Two dogs produced low levels (peak: $0.28 \pm 0.03$ ) and two high levels (peak: $0.46 \pm 0.02$ ).
} 


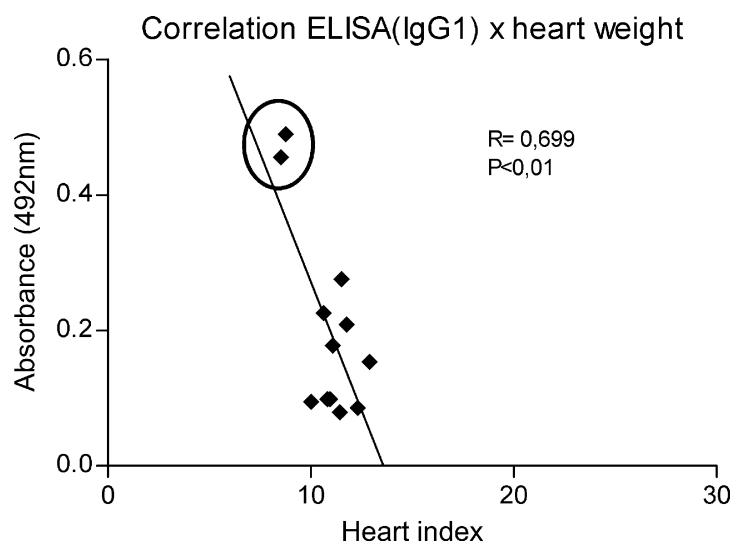

Fig. 2. Correlation analysis between heart index (heart weight/total body weight $\times 100$ ) and IgG1 production by ELISA realized 100 weeks post-infection in Beagle dogs infected with Y, Berenice-78 and ABC Trypanosoma cruzi strains. The two selected points indicate the animals that did not show cardiomegaly.

experimental models (Spinella et al., 1992; Giordanengo et al., 2000). While some studies did not detect differences between the levels of these immunoglobulins amongst individuals with different clinical manifestations (Cerban et al., 1993; Michailowsky et al., 2003), others have found higher levels of IgG2 antibodies in sera of patients with cardiac and digestive manifestations of the disease (Morgan et al., 1996; Cordeiro et al., 2001). Furthermore, the indeterminate form of Chagas disease was associated with high IgG1 levels (Cordeiro et al., 2001).

The non-stimulated PBMC proliferative response of infected animals showed a slight decreasing tendency at 10 weeks after infection (Fig. 3A). Accordingly, PBMC proliferation results described in literature have shown an immunosuppressive state, which is normally observed during the final of acute phase in T. cruzi infection (Barr et al., 1991). All infected animals showed the same pattern of proliferative response. The trypomastigote-induced PBMC proliferative response observed in infected animals showed significant difference $(P<0.05)$ to uninfected dogs 6 weeks after inoculation, showing a small decrease at the end of acute phase (10 weeks after infection) (Fig. 3B).

Furthermore, the high trypomastigote-induced PBMC proliferative responses observed in infected dogs presenting cardiomegaly were, also, related with low or lack IgG1 production (Table 1; Figs. 1-3). Animals that present high PBMC reactivity in vitro against $T$. cruzi antigens are likely to have larger number of T CD4 cell clones (Th1 phenotype) that produce principally inflammatory cytokines. The severity of the cardiac clinical forms of Chagas disease in patients has
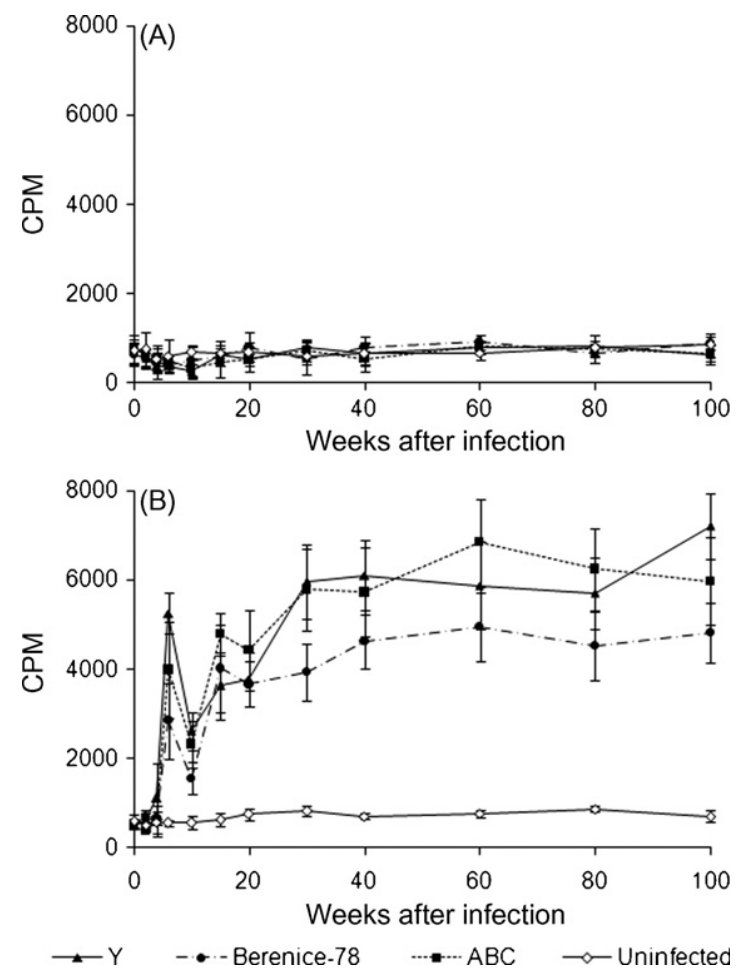

Fig. 3. In vitro PBMC proliferation of non-stimulated (A) and trypomastigote antigen-stimulated (B) before, during acute and chronic phases from Beagle dogs infected with the Y, Berenice-78 and ABC T. cruzi strains.

been associated with high PBMC reactivity to $T$. cruzi antigen (Gomes et al., 2003).

This study represented the first attempt to measure the kinetics of IgG isotype production by ELISA and FC in Beagle dogs infected by different $T$. cruzi strains. In conclusion, our results indicate that $T$. cruzi strains (Y, Berenice-78 and $\mathrm{ABC}$ ), which show distinct patterns of virulence and pathogenicity in dogs, stimulate different IgG1 levels and PBMC proliferation. We demonstrate, here, the correlation between low IgG1 levels, high PBMC proliferation and presence of cardiomegaly.

\section{Acknowledgments}

This work was supported by Fundação de Amparo à Pesquisa do Estado de Minas Gerais (FAPEMIG), Conselho Nacional de Pesquisa de Desenvolvimento Científico e Tecnológico (CNPq), and research fellowships from CNPq (Galvão, L.M.C., Afonso, L.C.C., Lana, M., Bahia, M.T., and Guedes, P.M.M. received doctoral fellowships). We also thank Lívia de Figueiredo and Geovam Crepalde from the Laboratório de Doença de Chagas, Instituto de Ciências Biológicas, UFOP, for animal care and Afonso da Costa Viana from 
the Departamento de Parasitologia, Instituto de Ciências Biológicas, UFMG, for cultivation and production of $T$ cruzi trypomastigotes forms.

\section{References}

Barr, S.C., Dennis, V.A., Klei, T.R., Norcross, N.L., 1991. Antibody and lymphoblastogenic responses of dogs experimentally infected with Trypanosoma cruzi isolates from North American mammals. Vet. Immunol. Immunopathol. 29 (3-4), 267-283.

Brener, Z., 1965. Comparative studies of different strains of Trypanosoma cruzi. Ann. Trop. Med. Parasitol. 59, 19-26.

Briere, F., Servet-Delprate, C.H., Bridon, J.M., 1994. Human interleukin 10 induces naves surface immunoglobulin D+ (slgD+) B cells to secrete IgG1 and IgG3. J. Exp. Med. 179, 757-762.

Cerban, F.M., Gea, S., Menso, E., Vottero-Cima, E., 1993. Chagas' disease: IgG isotypes against Trypanosoma cruzi cytosol acidic antigens in patients with different degrees of heart damage. Clin. Immunol. Immunopathol. 67, 25-30.

Cordeiro, F.D., Martins-Filho, O.A., Rocha, M.O.C., Adad, S.J., Correa-Oliveira, R., Romanha, A.J., 2001. Anti-Trypanosoma cruzi immunoglobulin G1 can be a useful tool for diagnosis and prognosis of human Chagas' disease. Clin. Diagn. Lab. Immunol. 1, 112-118.

Day, M.J., 2007. Immunoglobulin G subclass distribution in canine leishmaniosis: a review and analysis of pitfalls in interpretation. Vet. Parasitol. 20 (147), 2-8.

Deplazes, P., Smith, N.C., Arnold, P., Lutz, H., Eckert, J., 1995. Specific IgG1 and IgG2 antibody responses of dogs to Leishmania infantum and other parasites. Paras. Immunol. 17, 451-458.

Giordanengo, L., Fretes, R., Diaz, H., Cano, R., Bacile, A., VotteroCima, E., Gea, S., 2000. Cruzipain induces autoimmune response against skeletal muscle and tissue damage in mice. Muscle Nerve. 23, 1407-1413.

Gomes, J.A., Bahia-Oliveira, L.M., Rocha, M.O., Martins-Filho, O.A., Gazzinelli, G., Correa-Oliveira, R., 2003. Evidence that development of severe cardiomyopathy in human Chagas' disease is due to a Th1-specific immune response. Infect. Immun. 71, 1185-1193.

Guedes, P.M.M., Veloso, V.M.V., Tafuri, W.L., Galvão, L.M.C., Carneiro, C.M., Lana, M., Chiari, E., Soares, K.A., Bahia, M.T., 2002. The dog as model for chemotherapy of the Chagas' disease. Act. Trop. 84, 9-17.

Guedes, P.M.M., Veloso, V.M.V., Caliari, M.V., Carneiro, C.M., Souza, S.M., Lana, M., Chiari, E., Bahia, M.T., Galvão, L.M., 2007. Trypanosoma cruzi high infectivity in vitro is related to cardiac lesions during long-term infection in Beagle dogs. Mem. Inst. Oswaldo Cruz. 102, 141-147.
Kawano, Y., Noma, T., Yata, J., 1994. Regulation of human IgG subclass production by cytokines IFN- $\gamma$ and IL- 6 act antagonistically in the induction of human IgG1 but additively in the induction of IgG2. J. Immunol. 153, 4948-4958.

Lana, M., Chiari, C.A., 1986. Caracterização biológica comparativa das cepas Berenece-78 de Trypanosoma cruzi, isoladas da mesma paciente em diferentes períodos. Mem. Inst. Oswaldo Cruz 81, 247-253.

Lana, M., Chiari, E., Tafuri, W.L., 1992. Experimental Chagas'disease in dogs. Mem. Inst. Oswaldo Cruz 87, 59-71.

Martins-Filho, O.A., Pereira, M.E.S., Carvalho, J.F., Cançado, J.R., Brener, Z., 1995. Flow cytometry, a new approach to detect antilive trypomastigote antibodies and monitor the efficacy of specific treatment in human Chagas' disease. Clin. Diagn. Lab. Immunol. 2, 569-573.

Michailowsky, V., Luhrs, K., Rocha, M.O., Fouts, D., Gazzinelli, R.T., Manning, J.E., 2003. Humoral and cellular immune responses to Trypanosoma cruzi-derived paraflagellar rod proteins in patients with Chagas' disease. Infect Immun. 71, 3165-3171.

Morgan, J., Dias, J.C.P., Gontijo, E.D., Bahia-Oliveira, L., CorreaOliveira, R., Colley, D.G., Powell, M.R., 1996. Anti-Trypanosoma cruzi antibody isotype profiles in patients with different clinical manifestations of Chagas' disease. Am. J. Trop. Med. Hyg. 55, 355-359.

Oliveira, J.A., 1998. Heart aneurysm in Chagas' disease. Rev. Inst. Med. Trop. Sao Paulo 40, 301-307.

Quinnell, R.J., Courtenay, O., Garcez, L.M., Kaye, P.M., Shaw, M.A., Dye, C., Day, M.J., 2003. IgG subclass responses in a longitudinal study of canine visceral leishmaniasis. Vet. Immunol. Immunopathol. 91, 161-168.

Reed, S.G., Scott, P., 1993. T-cell and cytokine responses in leishmaniasis. Curr. Opin. Immunol. 5, 524-531.

Silva, L.H.P., Nussenzweig, V., 1953. Sobre uma cepa de Trypanosoma cruzi altamente virulenta para o camundongo branco. Folia Clin. Biol. 20, 191-203.

Snedecor, G.W., Cochran, W.G., 1989. Statistical Methods. Yowa State University Press, 289 pp.

Spinella, S., Liegeard, P., Hontebeyrie-Joskowicz, M., 1992. Trypanosoma cruzi: predominance of $\mathrm{IgG} 2 \mathrm{a}$ in nonspecific humoral response during experimental Chagas' disease. Exp. Parasitol. 74, $46-56$.

Tafuri, W.L., 1987. Patogenia da doença de Chagas. Rev. Inst. Med. Trop. São Paulo 29, 194-199.

Voller, A., Bidwell, D.E., Bartlett, A., 1976. Enzyme immunoassays in diagnostic medicine. Theory and practice. Bull. W.H.O. 53, 5565.

WHO, 2005. Tropical Disease Research: Progress 2003-2004 Seventeenth Programme Report of the UNICEF/UNDP/World Bank/ WHO. Programme Report n. 17, Geneva. 